\title{
AN EXTREMELY RARE PRESENTATION OF PRIMARY RETROPERITONEAL RETROVESICAL HYDATID CYST AS OBSTRUCTIVE UROPATHY
}

\author{
Rana Pratap Singh ${ }^{1}$, Nikhil Ranjan², Khalid Mahmood ${ }^{3}$, Ahsaan Ahmed ${ }^{4}$, Rajesh Tiwari $^{5}$
}

\author{
1 MCH Student, Department of Urology, IGIMS. \\ 2MCH Student, Department of Urology, IGIMS. \\ ${ }^{3}$ Assistant Professor, Department of Urology, IGIMS. \\ ${ }^{4}$ Assistant Professor, Department of Urology, IGIMS. \\ 5 Professor, Department of Urology, IGIMS.
}

\begin{abstract}
Primary retroperitoneal hydatid cyst is extremely rare and only few case reports exist in literature. We present a case report of retrovesical hydatid cyst mimicking retroperitoneal cystic tumor presenting as life threatening obstructive uropathy with raised serum creatinine. The patient was treated with initial bilateral percutaneous nephrostomy drainage and later cystectomy. The case report points to difficulty in diagnosis, problems in management and potentially life threatening nature of disease.
\end{abstract}

KEYWORDS: Retroperitoneal, Retrovesical, Hydatid Cyst, Obstructive Uropathy.

HOW TO CITE THIS ARTICLE: Rana Pratap Singh, Nikhil Ranjan, Khalid Mahmood, Ahsaan Ahmed, Rajesh Tiwari. "An Extremely Rare Presentation of Primary Retroperitoneal Retrovesical Hydatid Cyst as Obstructive Uropathy". Journal of Evolution of Medical and Dental Sciences 2015; Vol. 4, Issue 90, November 09; Page: 15596-15597, DOI: 10.14260/jemds/2015/2238.

CASE REPORT: A Thirty year old male patient presented with complains of poor flow for two years, suprapubic lump for last six months and fever for last one week. On examination the patient was anaemic, cachectic and febrile. A suprapubic lump was palpable, the lump was non-tender, smooth and cystic. On DRE prostate was normal and a cystic mass separate from it was felt .A foley's catheter was passed but lump did not subsided .Patient's Haemoglobin was 6.4 gm $\%$ and serum creatinine $7.4 \mathrm{mg} / \mathrm{dl}$. A NCCT showed a cystic structure behind the bladder with the bladder pushed up with bilateral hydroureteronephrosis (Figure 1).

A diagnosis of obstructive uropathy due to retoperitoneal cystic tumor was made. There were no cyst in the liver or lungs. Under ultrasound guidance bilateral percutaneous nephrostomies were performed. Purulent urine came out of both kidneys. The patient was treated with broad spectrum antibiotics for his fever. Even after two days of drainaige and urine becoming clear the fever failed to subside, a decision to puncture and aspirate the cyst to relieve the patient's uropathy was made. The cyst was punctured under aseptic conditions under ultrasound guidance. As soon as the cyst was punctured the patient developed chills and rigor and fever intensified and real time Ultrasound showed typical picture of hydatid cyst with "Water lily sign"with the endocyst floating free in the ectocyst (Figure 2). The aspirated fluid was purulent and sent for culture and sensitivity. A diagnosis of anaphylactic shock due to hyatid antigen was made and hydrocortisone was administered. The patient recovered from the acute episode and was kept under ICU overnight. Blood transfusions were given for correcting anemia.

Financial or Other, Competing Interest: None.

Submission 19-10-2015, Peer Review 20-10-2015,

Acceptance 27-10-2015, Published 09-11-2015.

Corresponding Author:

Dr. Rana Pratap Singh,

Lane 1B, Shivapuram, Vijaynagar,

Rukunpura, Patna, Bihar.

E-mail: ranapratapsingh02@gmail.com

DOI:10.14260/jemds/2015/2238.
The patients creatinine levels came down over two weeks and fever subsided. Patient was then posted for surgical removal of cyst. Abdomen was opened via infraumblical midline incision. Cyst was exposed posterior to bladder.

Cyst was removed in toto along with endocyst and ectocyst (Figure 3). Postoperative nephrostomogram showed good flow of contrast into bladder and subsequently PCNs were removed. The patient recovered well and was discharged, follow up after 6 months failed to show recurrence.

DISCUSSION: Hydatid disease is parasitic infestation caused by tapeworm E. granulosus. The most commonly involved organs are the liver and the lungs. After ingesting, the oncospheres hatch and transverse the intestinal wall, disseminating to the liver first, secondarily to lung, and finally anywhere to form unilocular cysts. Other pathogenic hypotheses for isolated retroperitoneal or retrovesical cysts have also been proposed.

A lymphatic route could lead the larvae from the intestinal lymph vessels to the thoracic channel and then anywhere in the body, or the embryo could remain in the rectal ampulla and migrate through the hemorrhoidal vessels to achieve a prerectal or retrovesical location and then develop. The classical radiological signs of hydatid cysts on CT are either a calcified rim mass or a multiseptated cyst; daughter cysts on CT scan are pathognomonic.

Lockhart and Sapinza first reported primary retroperinoeal hydatid cyst in 1958.(1) Occasional case reports from endemic areas appeared in english literature. Even when they present in retroperitoneum, retro vesical area is seldom involved (Approximately 13\%).(2) The hydatid cyst is certainly not the first differential diagnosis of isolated pelvic cyst in view of its rarity. The other differentials are diverticula bladder, cysts of seminal vesicle, cystic masses of sigmoid and rectum, ectopic kidney with hydronephrosis, lymphatic cysts, vascular aneurysms and pseudo-epithelial cysts.

Though the presence of daughter cysts on CT is pathognomonic, in some cases the final diagnosis may be made only after surgery. Real time ultra sound for hydatid gravels may be especially helpful. 
The pathogenesis in primary pelvic cyst is specially enigmatic, postulated is the rupture and release of liver hydatid with primary healed but this explanation is less than satisfactory. (3)

Retrovesical hydatid cysts may have varied and nonspecific presentation. In the series by Angulo et al.(3) the most common presentation was a palpable mass followed by flank pain, LUTS, urinary retention and dysuria.

Rare case reports of cyst causing ipsilateral renal loss are available, but cyst leading to acute kidney injury due to bilateral obstructive uropathy is not mentioned as a presentation except in a pediatric case.(4) In one case report hydatid cyst mimicked retrocaval ureter.(5) The incidence of anaphylaxis in hepatic hydatid disease varies and was noted in 3 of 20 patients $(15 \%)$ in one series.(6)

This case taught us many important lessons, no presentation is typical, hydatid cyst is capable of causing acute kidney injury by compressing the bladder base area and trigone from the retroperitoneum, CT SCAN may miss the diagnosis in atypical cases, howsoever rare the risk of anaphylaxis be, it is present and literature confirms the associated fatality in the absence of immediate intervention, Cyst puncture with aspiration of fluid, which is helpful in diagnosis, should be avoided as it may cause acute anaphylaxis. Preoperative Casoni, Weinberg, and hemagglutination tests were not performed because hydatid cyst was not suspected clinically or on radiological grounds.

\section{REFERENCES:}

1. Lockhart, J. and Sapinza, V. C.: Primary retroperitoneal hydatid cyst, Excerpta Med. (Surg). 12(2) 4513 p. 968, 1958.

2. Primary retroperitoneal hydatid cyst: report of 2 cases and review of 41 published cases. Akbulut S1, Senol A, Ekin A, Bakir S, Bayan K, Dursun M. Int Surg. 2010 Jul-Sep;95(3):189-96.

3. Isolated retrovesical and extrarenal retroperitoneal hydatidosis: clinical study of 10 cases and literature review Angulo JC, Escribano J, Diego A,

Sanchez-Chapado M. J Urol 1998; 159: 76-82.

4. Retrovesical Echinococcal Cyst Causing Renal Failure and Nocturnal Enuresis in a Child By Levent Emir, Cankon Germiyanog lu, Alpaslan Lokumcu, and Demokan Erol Ankara, Turkey.

5. Crundwell, M. C. and Blacklock, A. R.: Retroperitoneal hydatid cyst mimicking retrocaval ureter. $\mathrm{Br} \mathrm{J}$ Urol, 81: 168, 1998.

6. Pishori, T., Azami, R. and Ali, S. M.: Hydatidosis: experience with hepatic and pulmonary hydatid disease. J Pak Med Assoc,48: 205, 1998.

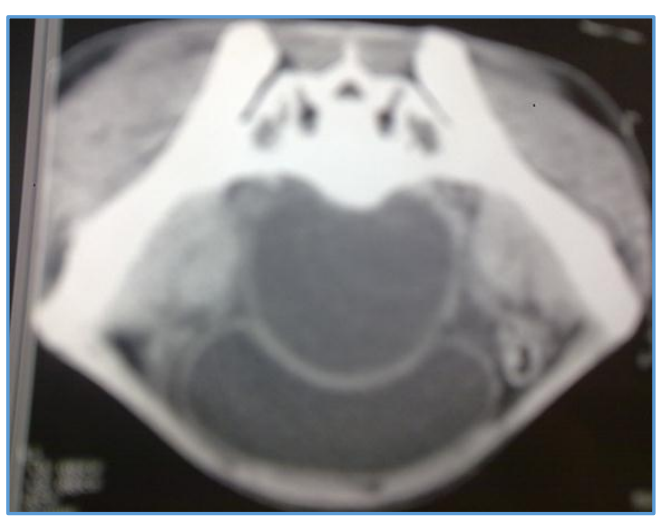

Fig. 1

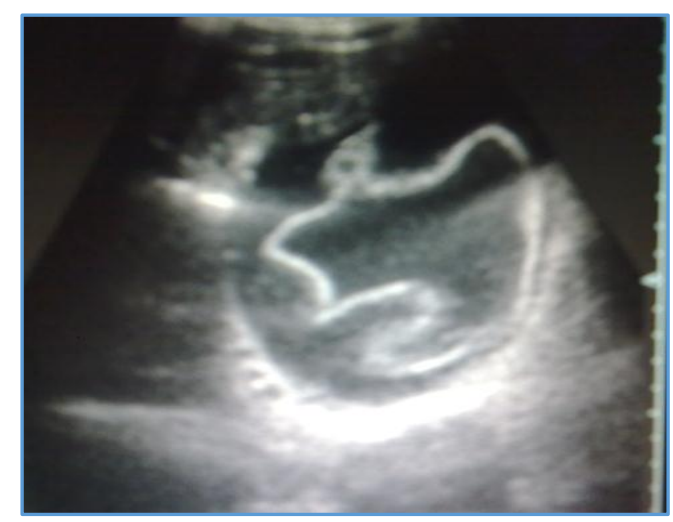

Fig. 2

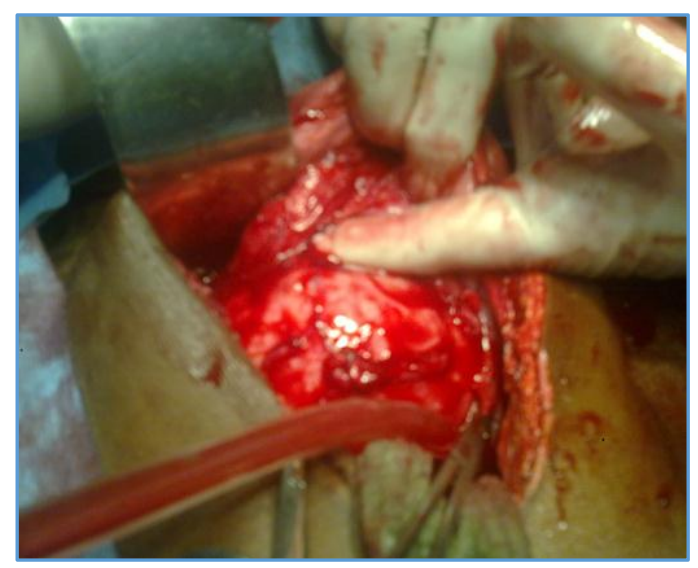

Fig. 3 